\title{
Clinical features and outcomes of PComA aneurysms originating from fetal posterior communicating arteries in a single institution
}

Xin Chen 1,2,3,4, Hao Li ${ }^{1,2,3,4}$, Ming-Ze Wang ${ }^{1,2,3,4}$, Mao-gui Li $i^{1,2,3,4}$, Yong Cao ${ }^{1,2,3,4}$, Dong Zhang ${ }^{1,2,3,4}$, Yan Zhang ${ }^{1,2,3,4}$, Hao Wang ${ }^{1,2,3,4^{*}}$ and Shuo Wang ${ }^{1,2,3,4^{*}}$

\begin{abstract}
Background: The aim of this study was to retrospectively analyze our experience with the patients who underwent surgical treatment of posterior communicating artery (PComA) aneurysms originating from fetal posterior cerebral artery (FPCA) and analyze the risk factors for the postoperative radiological infarction and outcome.

Methods: From 2011 to 2020, we retrospectively reviewed 74 PComA aneurysms originating from fPCA in terms of the clinical and radiological features and obtained the follow-up data from the Department of Neurosurgery, Beijing Tiantan Hospital, Capital Medical University. The relationships between these features and follow-up data were assessed with the univariate and multivariate analysis.

Results: In this series, 74 aneurysms were occurring at the origin of fPCAs. All the patients showed complete obliteration of their aneurysms. Full fPCA type tends to be a predictive factor for radiological infarction (univariate $X^{2}$ $=5.873, P=0.027$; multivariate $\mathrm{OR}=0.264, P=0.060$ ). Postoperative radiological infarction (univariate $X^{2}=12.611, P$ $=0.001$; multivariate $\mathrm{OR}=6.033, P=0.043$ ), rupture (univariate $X^{2}=4.514, P=0.047$; multivariate $\mathrm{OR}=57.966, P=$ 0.044 ), and hypertension (univariate $X^{2}=5.301, P=0.024$; multivariate $O R=24.462, P=0.029$ ) tend to be the independent predictive factors for poor prognosis at 3 months after discharge.

Conclusions: In conclusion, we report a series of patients harboring aneurysms originating from the fPCA. Surgical clipping is a reliable strategy. Full fPCA type is related to postsurgical infarction. Postoperative radiological infarction, rupture, and hypertension tend to be the independent predictive factor for poor prognosis at 3 months after discharge.
\end{abstract}

Keywords: Aneurysm, Fetal posterior cerebral artery, Clinical features, Outcome

\section{Background}

The posterior communicating artery (PComA) plays a vital role in physiologically mediating the anterior and posterior cerebral circulation and providing a facilitating channel for reversible flow to maintain adequate cerebral

\footnotetext{
* Correspondence: cmu990103@126.com; captain9858@vip.sina.com 'Department of Neurosurgery, Beijing Tiantan Hospital, Capital Medical University, Beijing 100070, China

Full list of author information is available at the end of the article
}

perfusion. In most cases, the posterior cerebral artery (PCA) receives the blood supply predominantly from the posterior circulation [1]. Yet, a fetal variant of PCA, named fetal PCA (fPCA), whose overall incidence has been reported 3-36\% among the whole population, either unilaterally or bilaterally, gets its predominant blood supply from anterior circulation [2-4]. An fPCA is called a full fPCA if the P1 segment is not visualized on computed tomography angiography (CTA), magnetic

C C The Author(s). 2020 Open Access This article is licensed under a Creative Commons Attribution 4.0 International License, which permits use, sharing, adaptation, distribution and reproduction in any medium or format, as long as you give appropriate credit to the original author(s) and the source, provide a link to the Creative Commons licence, and indicate if changes were made. The images or other third party material in this article are included in the article's Creative Commons licence, unless indicated otherwise in a credit line to the material. If material is not included in the article's Creative Commons licence and your intended use is not permitted by statutory regulation or exceeds the permitted use, you will need to obtain permission directly from the copyright holder. To view a copy of this licence, visit http://creativecommons.org/licenses/by/4.0/ The Creative Commons Public Domain Dedication waiver (http://creativecommons.org/publicdomain/zero/1.0/) applies to the data made available in this article, unless otherwise stated in a credit line to the data. 
resonance angiography (MRA), or after injection of contrast into the vertebral artery; a partial PPCA if the P1 segment is smaller than the PcomA; or an intermediate fPCA if the P1 segment is as large as the PcomA.

The internal carotid artery (ICA)-PComA junction is the favorite site for intracranial aneurysms, and approximately 20 to $30 \%$ of cerebral aneurysms locate here [5-7]. ICAPComA aneurysms, so-called PComA aneurysms, are aneurysms of the ICA occurring at the origin of the PComA [8]. Either surgical clipping or endovascular obliteration of PComA aneurysms has a big chance to inadvertently injury the PComA itself or adjacent perforating arteries, leading to ischemic injury to dependent regions $[9,10]$. Especially in the cases of fetal variant circulation, the occlusion of the dominant feeder to these regions can be especially crippling, which will cause potential infarction of the midbrain, thalamus, and occipital region [5, 11, 12]. However, there were only few reports referring to surgical experiences about the PPCA aneurysms. In addition, the risk factors for the outcomes of surgical treatment for fPCA aneurysms have not been evaluated yet. Thus, the aim of this study was to retrospectively report our surgical experience with a series of 74 fPCA aneurysm patients in our institution and analyze the risk factors for the outcome.

\section{Methods}

\section{Patient population and follow-up data}

A total of 388 cases of PcomA aneurysms underwent microsurgery in the Department of Neurosurgery, Beijing Tiantan Hospital, Capital Medical University, from 2011 to 2020. Among these patients, 99 patients were identified with fPCA in the circle of Willis. Seventy-four out of 99 patients had their aneurysms originating from the fPCA. Clinical data were collected from the hospital records. The radiological data (including CT, CTA, and DSA) were examined on Picture Archiving and Communication Systems (PACS). Follow-up data were obtained from telephone reviews. This study was approved by the institutional review board in our hospital. All procedures performed in studies involving human participants were in accordance with the ethical standards of the institutional and/or national research committee and with the 1964 Helsinki Declaration and its later amendments or comparable ethical standards.

\section{fPCA identification and classification}

Generally speaking, the PPCA variant was defined as one with primary perfusion of the PCA vessels from ICA angiography injections and minimal perfusion of PCA vessels on vertebrobasilar angiography, often with an absent or hypoplastic P1 segment. Due to the architecture, fPCA was classified into two sorts, partial fPCA and full fPCA. For partial fPCA, P1 segment was hypoplastic and the PComA was significantly larger or the same in diameter (Fig. 1a, b), but for the regular PCA/PComA, P1 segment is significantly larger than PComA in diameter; full fPCA was defined by that the P1 segment of PCA was absent and the PComA was completely originating from ICA (Fig. 1c, d) [13]. The fPCA was determined by DSA or CTA (the patients without DSA).

\section{Complications and outcomes}

The main complication in this study was postoperative radiological infarction. Comparing with preoperative images on CT scan, the new infarction on the brain territory supplied by PCA was defined as postoperative radiological infarction. The Glasgow Outcome Scale (GOS) score of 3 months after discharge was assessed. According to the score, the GOS was classified into good outcome (GOS score was 4 and 5) and bad outcome (GOS score was 1, 2, and 3).

\section{Statistical analysis}

All data were analyzed by the use of SPSS statistical program (version 19.0 for Windows). The associations between clinical data and postoperative radiological infarction or outcome at 3 months after surgery were examined by Student's $t$ test for continuous variables and Pearson's $\chi^{2}$ test or Fisher's exact test for categorical variables. Then, the statistically significant parameters were enrolled into the binary logistic regression analysis to calculate the independent predictive factors. $P<0.05$ was considered statistically significant.

\section{Results}

\section{Clinical and radiological data}

The clinical features of the 74 patients are summarized in Table 1. The mean age of the patients was 57.7 years (range, 24-77). There was a female preponderance with the ratio of 56/18 (female/male).

The mean maximal aneurysm diameter was $7.34 \mathrm{~mm}$ (range, 3-25 $\mathrm{mm}$ ), and the medium type make up the vast majority (53/71.6\%). Thirty-nine (52.7\%) aneurysms showed regular sacciform shape, and others were irregular (lobulated or rectangular). Thirty-one $(41.9 \%) \mathrm{pa}$ tients showed Bilateral fPCA type, and 16 (21.6\%) and 27 (36.5\%) showed left and right side type, separately. The full fPCA type accounts for 45 (60.8\%) of all. Seventeen patients $(23.0 \%)$ had multiple aneurysms (the other aneurysms were located at ICA, middle cerebral artery, and anterior communicating artery) on preoperative angiography. Of all the patients, 9 (12.2\%) patients underwent temporary clipping. Sixteen (21.6\%) patients suffered from the third cranial nerve palsies, and 46 $(62.2 \%)$ had the case history of hypertension. The preoperative CT scan revealed 48 (64.9\%) subarachnoid hemorrhage (SAH), with Hunt-Hess grade I of 25 (33.8\%), II of 14 (18.9\%), and III of 9 (12.2\%). 

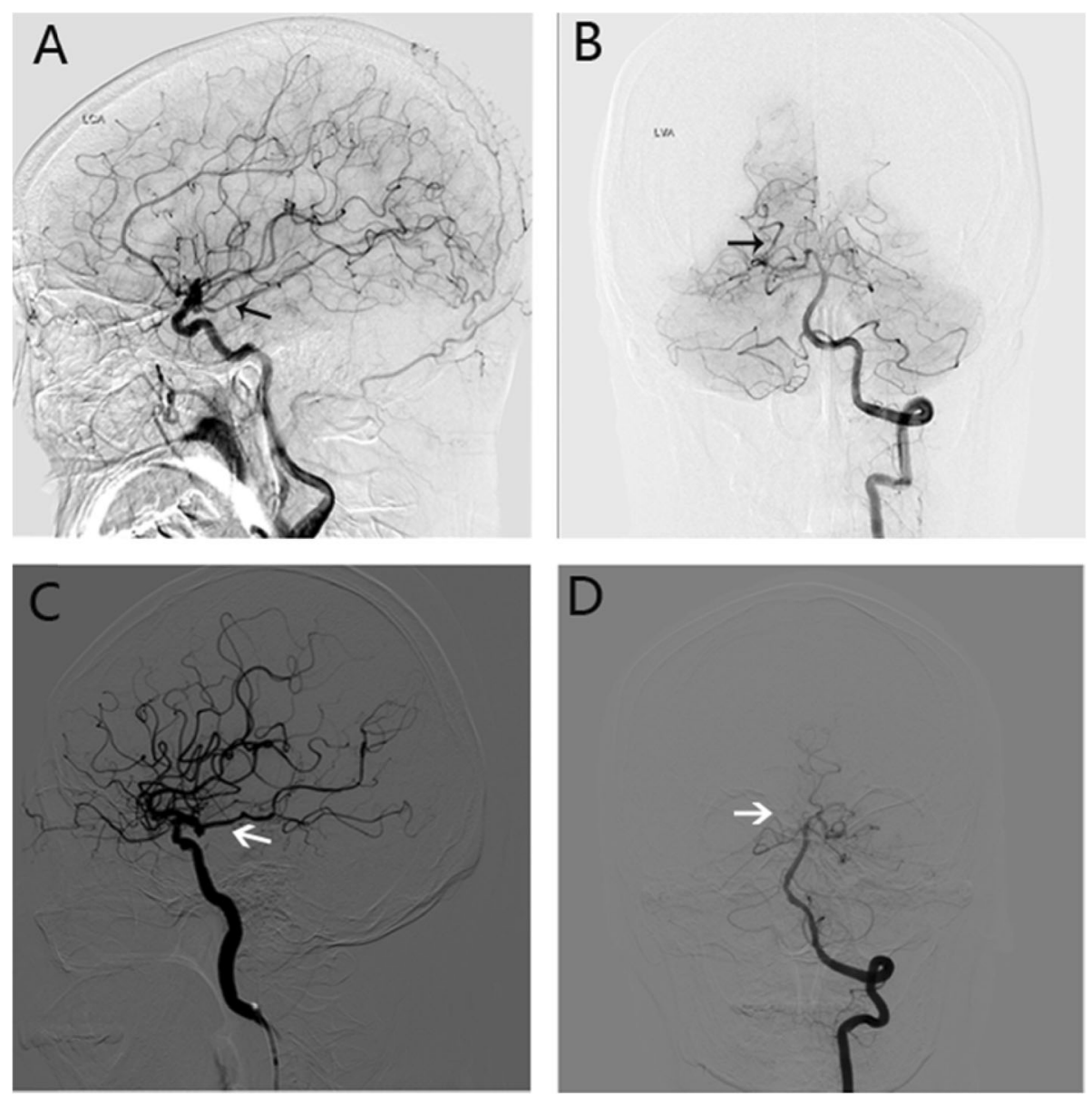

Fig. 1 DSA demonstrating the partial fPCA and complete fPCA. a, b Representative images of partial fPCA on DSA. The arrow on a shows the PComA is continuous as PCA in the same diameter of the lumen, but the arrow on $\mathbf{b}$ shows the PCA is originating from the basilar artery. $\mathbf{c}, \mathbf{d}$ Representative images of complete PPCA on DSA. The arrow on $\mathbf{c}$ shows PComA is continuous as PCA in the same diameter of the lumen; the arrow on $\mathbf{d}$ shows the right PComA was absent. FPCA, fetal posterior cerebral artery; PComA, posterior communicating artery; PCA, posterior cerebral artery; DSA, digital subtraction angiography

\section{Radiological infarction and outcomes}

All the patients were identified getting complete obliterations of their aneurysms and having preservation of fPCA by postoperative DSA or CTA (Fig. 2a-d). Based on the postoperative CT scan before the patients were discharged, 19 patients (25.7\%) showed radiological infarction on the PCA supplying territory (Fig. 2e-h). GOS scores for the 74 patients at the time of 3 months after discharge are shown in Table 2. Most patients (85.1\%) have a GOS score of 4 and 5 defined as good outcome.

\section{Predictors of postoperative radiological infarction and poor functional outcome}

For postoperative radiological infarction, aneurysm size $(t=-2.482, P=0.015)$, fPCA type $\left(\chi^{2}=5.873\right.$, $P=0.027)$, and intraoperative temporary clipping $\left(\chi^{2}\right.$ $=4.794, P=0.043$ ) showed dependencies.
For the outcome of 3 months after discharge, aneurysm size $(t=-2.492, P=0.016)$, age $(t=-$ 2.195, $P=0.031)$, hypertension $\left(\chi^{2}=5.301, P=0.024\right)$, rupture $\left(X^{2}=4.514, P=0.047\right)$, and postoperative radiological infarction $\left(\chi^{2}=12.611, P=0.001\right)$ were significantly related. The binary logistic regression analysis revealed that postoperative radiological infarction (odds ratio $(\mathrm{OR})=6.033,95 \%$ confidence interval $(\mathrm{CI})$ (1.057-34.437), $P=0.043)$, rupture $(\mathrm{OR}=57.966$, 95\% CI (1.112-3020.66), $P=0.044)$, and hypertension (OR $=24.462,95 \%$ CI $(1.385-431.917), P=0.029)$ are the potential independent predictive factors (Table 3 ).

\section{Discussion}

Morphology and hemodynamics have been revealed to play important roles in the generation and rupture of intracranial aneurysms [14]. As reported, the fPCA is a common variant of Willis circulation in the whole population $[1,2,15,16]$. Generally speaking, fPCA contributes to 
Table 1 Summary of clinical data of 74 patients

\begin{tabular}{|c|c|c|}
\hline \multicolumn{2}{|l|}{ Demographics } & \multirow{2}{*}{$\frac{\text { No. of cases }(\%)}{18(24.3 \%)}$} \\
\hline Sex & Male & \\
\hline & Female & $56(75.7 \%)$ \\
\hline \multirow[t]{2}{*}{ Age in years } & $<60$ years & $39(52.7 \%)$ \\
\hline & Age $\geq 60$ years & $35(47.3 \%)$ \\
\hline \multirow[t]{2}{*}{ Hypertension } & Yes & $46(62.2 \%)$ \\
\hline & No & $28(37.8 \%)$ \\
\hline \multirow[t]{4}{*}{ Hunt-Hess grade } & $0^{*}$ & $26(35.1 \%)$ \\
\hline & 1 & $25(33.8 \%)$ \\
\hline & 2 & $14(18.9 \%)$ \\
\hline & 3 & $9(12.2 \%)$ \\
\hline \multirow[t]{2}{*}{ Third cranial nerve palsies } & Yes & $16(21.6 \%)$ \\
\hline & No & $58(78.4 \%)$ \\
\hline \multirow[t]{2}{*}{ fPCA type } & Partial & 29 (39.2\%) \\
\hline & Full & $45(60.8 \%)$ \\
\hline \multirow[t]{3}{*}{ fPCA sides } & Bilateral & $31(41.9 \%)$ \\
\hline & Left side & $16(21.6 \%)$ \\
\hline & Right side & $27(36.5 \%)$ \\
\hline \multirow[t]{4}{*}{ Aneurysm size } & Small (diameter < $0.5 \mathrm{~cm}$ ) & $15(20.3 \%)$ \\
\hline & Medium $(0.5 \mathrm{~cm} \leq$ diameter $<1.5 \mathrm{~cm})$ & $53(71.6 \%)$ \\
\hline & Large $(1.5 \mathrm{~cm} \leq$ diameter $<2.5 \mathrm{~cm})$ & $5(6.8 \%)$ \\
\hline & Giant (diameter $\geq 2.5 \mathrm{~cm}$ ) & $1(1.4 \%)$ \\
\hline \multirow[t]{2}{*}{ Aneurysm shape } & Regular & $39(52.7 \%)$ \\
\hline & Irregular & $35(47.3 \%)$ \\
\hline \multirow[t]{2}{*}{ Multiple aneurysms } & Yes & $17(23.0 \%)$ \\
\hline & No & $57(77.0 \%)$ \\
\hline \multirow[t]{2}{*}{ Intraoperative temporary clipping } & Yes & $9(12.2 \%)$ \\
\hline & No & $65(87.8 \%)$ \\
\hline \multirow[t]{2}{*}{ Postoperative radiological infarction } & Yes & $19(25.7 \%)$ \\
\hline & No & $55(74.3 \%)$ \\
\hline \multirow[t]{2}{*}{ Postoperative complications } & Yes & $24(32.4 \%)$ \\
\hline & No & $50(67.6 \%)$ \\
\hline
\end{tabular}

*Hunt-Hess grade 0 refers to unruptured aneurysms

a particular architecture of Willis circle and is more likely to gestate aneurysms due to disturbed hemodynamics distribution [17, 18]. In a comparative analysis about PComA, Thiarawat et al. revealed that ipsilateral fPCAs get a higher possibility to harbor aneurysms than other hemodynamics types ( $42 \%$ vs $14 \%, P<0.001$ ) [19]. Similarly, in this series, we found the fPCA aneurysm patients accounted for $19.1 \%$ (74 out of 388) of the population with PComA aneurysms.

The ipsilateral fPCA feeds the whole territory, which is formerly supplied by conventional posterior cerebral artery, and exposes to more violent impact of blood flow. In this case, aneurysms grow more easily. In case of blocking or injury of fPCA, infarction develops and patients tend to suffer from poor outcomes because of the lack of compensatory from posterior cerebral circulation [20]. Cerebral infarction is strongly correlated with angiographic vasospasm for ruptured aneurysms. The constituent and accompanying inflammatory could cause a strong constriction of the blood vessels. But in some cases, angiographic vasospasm was not detected. It is because that there were still other reasons, such as small-vessel spasm and micro-thromboembolism, cortical spreading ischemia, intraoperative hypotension, coagulopathy, perforator occlusion attributable to the aneurysmsecuring procedure, or a complication of catheter 

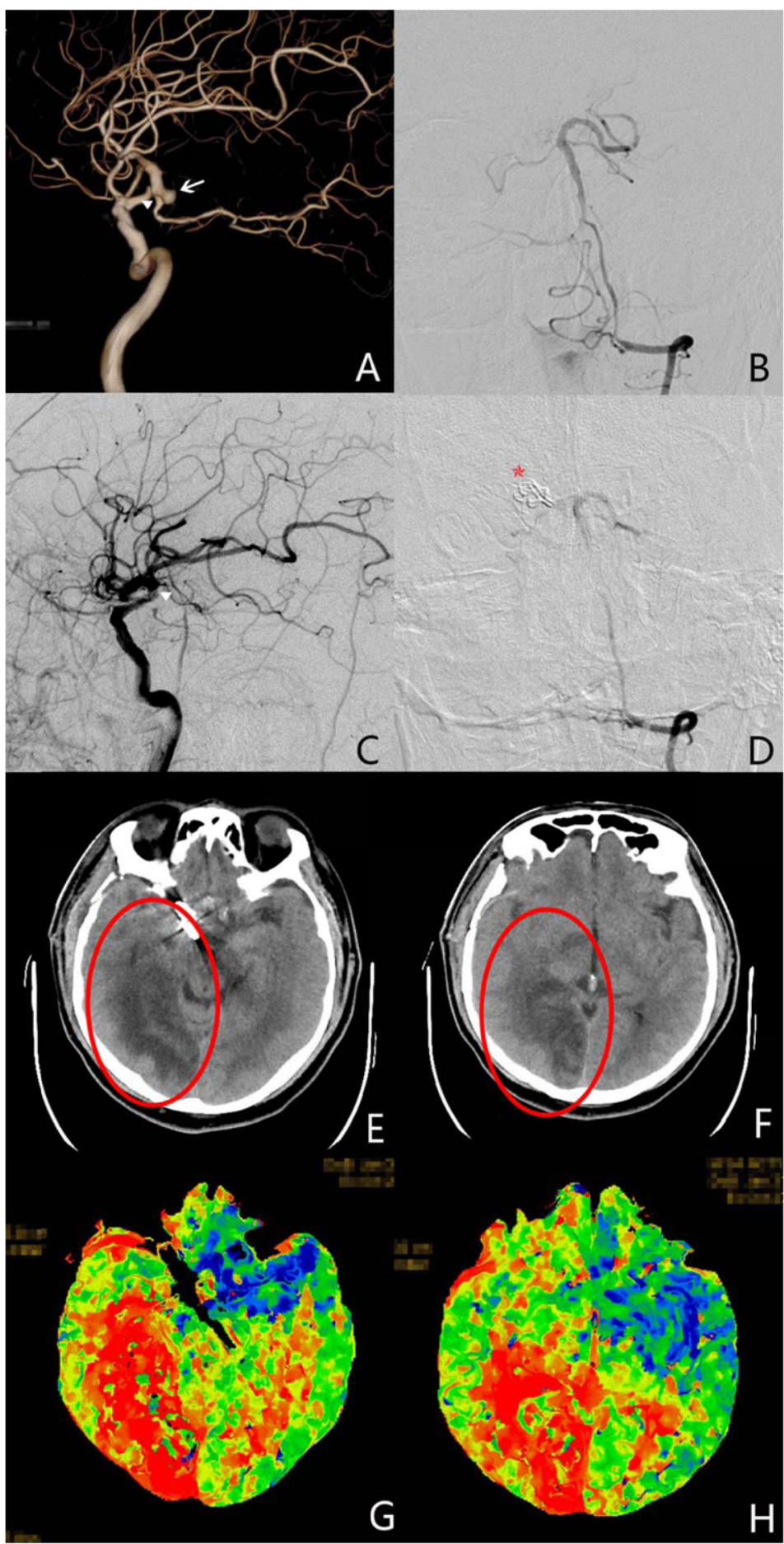

Fig. 2 The demonstration of a male, 55-year-old case. a The 3D RCA development of DSA shows the PComA aneurysm originating from the origin of the full-type PCA. White arrowhead shows the aneurysm. The triangle shows the origin of full PCA type from RCA system. $\mathbf{b}$ The vertebral development of DSA shows the missing of PCA. $\mathbf{c}$ The postoperative RCA development of DSA shows no image of the PCA after surgical clipping and the aneurysm is totally eliminated. The triangle shows the preoperative origin site of full PCA type from RCA system. $\mathbf{d}$ The postoperative vertebral development of DSA shows the missing of PCA and totally occlusion of the aneurysm for $\mathbf{c}$. Five-pointed star indicates the titanium clips. e, $\mathbf{f}$ The postoperative CT scan show the infarction of right temporal and occipital lobe. The red ellipses demonstrate the ischemic low density. $\mathbf{g}, \mathbf{h}$ The CT perfusion imaging (axial time to peak) shows the ischemia (red area) of right temporal and occipital lobe 
Table 2 The prognosis of the 74 patients at 3 months after

\begin{tabular}{ll} 
surgery & No. of cases (\%) \\
\hline 5 GOS score & $51(68.9 \%)$ \\
4 (good) & $12(16.2 \%)$ \\
3 (pood) & $8(10.8 \%)$ \\
2 (poor) & $2(2.7 \%)$ \\
1 (poor) & $1(1.4 \%)$ \\
\hline
\end{tabular}

angiography. Therefore, in order to prevent the occurrence of postoperative cerebral infarction, blood pressure should be controlled steadily throughout the perioperative period to ensure effective cerebral perfusion pressure, careful handling should be recommended to protect perforating blood vessels during operation, and hemostatic agents should be used cautiously.

In this study, we described clinical data of 74 aneurysms in the origin site of posterior cerebral artery firstly (Table 1). We noticed some common features as aneurysms of other types, such as a female preponderance (56 vs 18) [21, 22], high prevalence of hypertension $(62.2 \%)$ [23], and high rupture rate, which are in accordance with the previous reports.

The architecture of FPCA is easier to cause hemodynamic aberrance. Due to the autocephalous architecture, injury or occlusion of the fPCA may bring about severe occipital infarcts and subsequent clinical complications, such as homonymous hemianopsia, alexia, aphasia, and hemiachromatopsia [16]. As suggested by the previous study, endovascular occlusion of an aneurysm and its parent artery is of high risk for patients with fetal-type PCA aneurysms [24]. In this surgical series of 74 cases, 19 (25.7\%) patients presented postoperative radiological infarction. Of them, 14 are from the perforator PComA, 2 have distal PCA infarct, and 3 share both. There are 4 that refer to MCA or ACA territory infarcts in this series. The univariate analysis $\left(\chi^{2}=5.873, P=0.027\right)$ and binary logistic regression $(\mathrm{OR}=0.264,95 \% \mathrm{CI}(0.066-1.056)$, $P=0.060$ ) demonstrated that full type of fPCA tend to be a predictive factor for postoperative radiological infarction.

Endovascular embolization of the PComA aneurysms has been widely accepted as an effective treatment modality. But endovascular treatment of fetal PComA faces some challenges, specifically for the ruptured ones. Roy et al. reported an independent association between incomplete occlusion and PPCA configuration [25]. Wallace et al. reported a complication rate of $14 \%$ and overall complete occlusion rate of $33 \%$ [26]. In this series, all aneurysms were completely clipped and 11 (15.0\%) suffered complications.

The follow-up data at 3 months after surgery showed that 63 patients $(85.1 \%)$ got favorable prognosis with the GOS of 4 and 5 . The regression analysis showed that postoperative radiological infarction, rupture, and hypertension tend to be the independent predictive factor for poor prognosis at 3 months after discharge.

Our study has some limitations. First, this study is a retrospective study and the number of patients is small, thus leading to bias. Prospective and a larger number of patients are needed to confirm the results. Second, the patients are screened in one institution and selection bias may have affected the veracity of outcome.

\section{Conclusion}

In conclusion, we report a series of patients harboring aneurysms originating from the PPCA. Surgical clipping is a reliable strategy. Patients with full fPCA type of PComAs got preference to develop postoperative radiological infarction. And postoperative radiological infarction, rupture, and hypertension history predicted poor prognosis at 3 months after surgeries.

Table 3 Univariate and multivariate analysis for postoperative RI and outcome of fPCA aneurysm

\begin{tabular}{|c|c|c|c|c|c|}
\hline \multirow[t]{2}{*}{ Dependent variable } & \multirow[t]{2}{*}{ Independent variable } & \multicolumn{2}{|c|}{ Univariate } & \multicolumn{2}{|c|}{ Multivariate } \\
\hline & & $P$ value & $t / x^{2}$ & $P$ value & Odds ratio $(95 \% \mathrm{Cl})$ \\
\hline \multirow[t]{3}{*}{ Radiological infarct } & Aneurysm size & $0.015^{*}$ & -2.482 & 0.214 & $1.096(0.949-1.265)$ \\
\hline & fPCA type & 0.027 & 5.873 & 0.06 & $0.264(0.066-1.056)$ \\
\hline & Intraoperative temporary clipping & $0.043^{\&}$ & 4.794 & 0.193 & $0.343(0.068-1.719)$ \\
\hline \multirow[t]{5}{*}{ 3-month outcome } & Aneurysm size & $0.016^{*}$ & -2.492 & 0.056 & $1.235(0.995-1.532)$ \\
\hline & Age & $0.031^{*}$ & -2.195 & 0.128 & $1.104(0.972-1.255)$ \\
\hline & Hypertension & $0.024^{\&}$ & 5.301 & 0.029 & $24.462(1.385-431.917)$ \\
\hline & Rupture & $0.047^{\&}$ & 4.514 & 0.044 & $57.966(1.112-3020.66)$ \\
\hline & Postoperative radiological infarction & $0.001^{\&}$ & 12.611 & 0.043 & $6.033(1.057-34.437)$ \\
\hline
\end{tabular}

${ }^{*} t$ test

${ }^{\&}$ Fisher's exact test 


\section{Abbreviations}

fPCA: Fetal posterior cerebral artery; PComA: Posterior communicating artery; ICA: Internal carotid artery; CT: Computerized tomography;

CTA: Computerized tomography angiography; CTP: CT perfusion imaging: DSA: Digital subtraction angiography; OR: Odds ratio; Cl: Confidence interval

\section{Acknowledgements}

Not applicable.

\section{Authors' contributions}

Design of the study: S.W., H.W., and X.C. Collection and writing the manuscript: X.C. and H.L. Analysis and interpretation of the data: X.C., MZ.W. and MG.L. Performing operations and modifying the manuscript: Y.C., D.Z., and Y.Z. The authors read and approved the final manuscript.

\section{Funding}

This research was supported by the following funds:

Special Fund for excellent talents of Dongcheng District of Beijing FY 2018, Principal: X.C.

National Natural Science Foundation (81671129), Principal: S.W.

\section{Availability of data and materials}

Please contact author for data requests.

\section{Ethics approval and consent to participate}

All procedures performed in studies involving human participants were in accordance with the ethical standards and approved by the Ethics Committee of Beijing TianTan Hospital, Capital Medical University (KY 2017012-02)

\section{Consent for publication}

Not applicable.

\section{Competing interests}

The authors declare that they have no competing interests.

\section{Author details}

'Department of Neurosurgery, Beijing Tiantan Hospital, Capital Medical University, Beijing 100070, China. ${ }^{2}$ China National Clinical Research Center for Neurological Diseases, Beijing 100070, China. ${ }^{3}$ Beijing Key Laboratory of Translational Medicine for Cerebrovascular Diseases, Beijing 100070, China. ${ }^{4}$ Center of Stroke, Beijing Institute for Brain Disorders, Beijing 100070, China.

Received: 14 March 2020 Accepted: 30 April 2020

Published online: 01 July 2020

\section{References}

1. Bisaria KK. Anomalies of the posterior communicating artery and their potential clinical significance. J Neurosurg. 1984;60(30):572-6.

2. Horikoshi T, Akiyama I, Yamagata Z, Sugita M, Nukui H. Magnetic resonance angiographic evidence of sex-linked variations in the circle of willis and the occurrence of cerebral aneurysms. J Neurosurg. 2002;96(4):697-703.

3. Schomer DF, Marks MP, Steinberg GK, Johnstone IM, Boothroyd DB, Ros $M R$, et al. The anatomy of the posterior communicating artery as a risk factor for ischemic cerebral infarction. N Engl J Med. 1994;330(220):1565-70.

4. Kameyama M, Okinaka SH. Collateral circulation of the brain with specia reference to atherosclerosis of the major cervial and cerebral arteries. Neurology. 1963;13:279-86.

5. O'Shaughnessy BA, Getch CC, Bendok BR, Batjer HH. Surgical management of unruptured posterior carotid artery wall aneurysms. Neurosurg Focus. 2003;15(1):1-8.

6. Pikus HJ, Heros RC. Surgical treatment of internal carotid and posterior communicating artery aneurysms. Neurosurg Clin N Am. 1998:9(4):785-95.

7. Rhoton AL Jr. Aneurysms. Neurosurgery. 2002;51(4 Suppl):S121-58.

8. Nery B, Araujo R, Burjaili B, Smith TR, Rodrigues JC Jr, Silva MN. "True" posterior communicating aneurysms: three cases, three strategies. Surg Neurol Int. 2016;7:1.

9. Lee KD, Kwon SC, Muniandy S, Park ES, Sim HB, Lyo IU. Tuberothalamic artery infarction following coil embolization of a ruptured posterior communicating artery aneurysm belonging to a transitional type posterior cerebral artery. A case report. Interv Neuroradiol. 2013;19(3):306-12.
10. Maeshima S, Ueyoshi A, Tsuura M, Takemoto H, Itakura T, Yoshida M, et al. Transient aphasia and persistent amnesia after surgery for internal carotid artery - posterior communicating artery aneurysm. J Clin Neurosci. 2002; 9(6):710-3.

11. Lee KC, Lee KS, Shin YS, Shin YS, Lee GW, Chung SK. Surgery for posterior communicating artery aneurysms. Surg Neurol. 2003:59(2):107-13.

12. Lambert SL, Williams FJ, Oganisyan ZZ, Branch LA, Mader EC Jr. a. Fetal-type variants of the posterior cerebral artery and concurrent infarction in the major arterial territories of the cerebral hemisphere. J Investig Med High Impact Case Rep. 2016;4(3):2324709616665409.

13. Shaban A, Albright KC, Boehme AK, Martin-Schild S. Circle of Willis variants: fetal PCA. Stroke Res Treat. 2013;105937:2013.

14. Chung BJ, Doddasomayajula R, Mut F, Detmer F, Pritz MB, Hamzei-Sichani F, et al. Angioarchitectures and hemodynamic characteristics of posterior communicating artery aneurysms and their association with rupture status. AJNR Am J Neuroradiol. 2017;38(11):2111-8.

15. Gibo H, Lenkey C, Rhoton AL Jr. Microsurgical anatomy of the supraclinoid portion of the internal carotid artery. J Neurosurg. 1981;55(4):560-74.

16. Jongen JC, Franke CL, Soeterboek AA, Versteege CW, Ramos LM, van Gijn J. Blood supply of the posterior cerebral artery by the carotid system on angiograms. J Neurol. 2002;249(4):455-60.

17. He Z, Wan Y. Is fetal-type posterior cerebral artery a risk factor for intracranial aneurysm as analyzed by multislice CT angiography. Exp Ther Med. 2018;15(1):838-46.

18. Lochner P, Golaszewski S, Caleri F, Ladurner G, Tezzon F, Zuccoli G, et al. Posterior circulation ischemia in patients with fetal-type circle of Willis and hypoplastic vertebrobasilar system. Neurol Sci. 2011;32(6):1143-6.

19. Thiarawat P, Jahromi BR, Kozyrev DA, Intarakhao P, Teo MK, ChoqueVelasquez J, et al. Are fetal-type posterior cerebral arteries associated with an increased risk of posterior communicating artery aneurysms. Neurosurgery. 2019;84(6):1306-12.

20. Li M, Wu J, Chen X, Jiang P, Yang F, Ma Y, et al. Symptomatic and silent cerebral infarction following surgical clipping of unruptured intracranial aneurysms: incidence, risk factors, and clinical outcome. Neurosurg Rev. 2018:41(2):675-82

21. Matsumura H, Kato N, Fujiwara Y, Hosoo H, Yamazaki T, Yasuda S, et al. Endovascular treatments for posterior cerebral artery aneurysms and vascular insufficiency of fetal-type circulation after parent artery occlusion. J Clin Neurosci. 2016:32:41-6.

22. Teng $H$, Wang $D$. Association between anatomical variations of the posterior communicating artery and the presence of aneurysms. Neurol Res. 2016;12:1-7.

23. Lindgren AE, Kurki MI, Riihinen A, Koivisto T, Ronkainen A, Rinne J, et al. Hypertension predisposes to the formation of saccular intracranial aneurysms in 467 unruptured and 1053 ruptured patients in Eastern Finland. Ann Med. 2014:46(3):169-76.

24. Xu J, Xu L, Wu Z, Chen X, Yu J, Zhang J. Fetal-type posterior cerebral artery: the pitfall of parent artery occlusion for ruptured P2 segment and distal aneurysms. J Neurosurg. 2015;123(4):906-14.

25. Roy AK, Howard BM, Haussen DC, Osbun JW, Halani SH, Skukalek SL, et al. Reduced Efficacy of the Pipeline Embolization Device in the Treatment of Posterior Communicating Region Aneurysms With Fetal Posterior Cerebral Artery Configuration. Neurosurgery. 2018;82(5):695-700.

26. Wallace AN, Kayan Y, Austin MJ, Delgado Almandoz JE, Kamran M, Cross DT 3rd, Moran CJ, et al. Pipeline Embolization of Posterior Communicating Artery Aneurysms Associated With a Fetal Origin Posterior Cerebral Artery. Clin Neurol Neurosurg. 2017;160:83-7.

Ready to submit your research? Choose BMC and benefit from:

- fast, convenient online submission

- thorough peer review by experienced researchers in your field

- rapid publication on acceptance

- support for research data, including large and complex data types

- gold Open Access which fosters wider collaboration and increased citations

- maximum visibility for your research: over $100 \mathrm{M}$ website views per year

At BMC, research is always in progress.

Learn more biomedcentral.com/submissions 